\title{
EFECTOS DE LAS VARIABLES AMBIENTALES EN LA ESTIMACIÓN DE MATERIA ORGÁNICA DEL SUELO A ESCALA REGIONAL EN UN AMBIENTE SEMIARIDO (REGIÓN DE MURCIA, ESPAÑA)
}

\author{
Pedro Pérez Cutillas ${ }^{1,2}$ \\ Gonzalo G. Barberá2 \\ Carmelo Conesa García ${ }^{1}$ \\ 1Departamento de Geografía. Universidad de Murcia. pedrope@um.es, cconesa@um.es \\ ${ }^{2}$ Grupo de Erosión y Conservación de Suelos. Centro de Edafología y Biología Aplicada del Segura, Consejo Superior de \\ Investigaciones Cientificas (CEBAS-CSIC). perezcutillas@cebas.csic.es, gbarbera@cebas.csic.es
}

\section{RESUMEN}

La estimación de valores edáficos mediante el empleo de técnicas de modelización espacial, basada en procesos y variables ambientales, es utilizada en este trabajo para analizar el efecto que tienen estas variables sobre la materia orgánica del suelo (MOS). La discusión es enormemente interesante por las conclusiones que se pueden extraer de las distintas implicaciones que ejercen los patrones ecológicos en su distribución y variabilidad espacial. En este estudio se ha utilizado una base de datos de 1126 muestras de materia orgánica en capa arable del suelo en la Región de Murcia (SE de España), con el fin de estimar el contenido de la MOS a escala regional (más de $10000 \mathrm{~km}^{2}$ ) mediante el uso de 21 variables ambientales como predictores. Entre las variables más influyentes destacan las asociadas al relieve (i.e. la pendiente, las curvaturas del terreno), la acumulación de flujo vinculada a las redes de drenaje, la precipitación, la radicación solar y ciertos índices específicos obtenidos mediante técnicas de teledetección, como el NDVI o el Índice Mineralógico de Arcillas CMI.

Palabras clave: Propiedades del suelo, materia orgánica del suelo, modelización, SIG, variables ambientales, teledetección. 


\section{ABSTRACT}

The estimation of soil values by means of spatial modeling techniques, based on environmental processes and variables, is used in this work to analyze the effect of these variables on soil organic matter (SOM). Discussing is extremely interesting conclusions can be extracted from the different implications on ecological patterns in the distribution and spatial variability. A database of 1,126 samples of organic matter in the arable layer from the Murcia Region (SE Spain) has been utilized to estimate the SOM contents at the regional scale (more than 10,000 km2), by using 21 environmental variables as predictors. Among the most influential variables are those related to relief (i.e. the slope and terrain curvature), flow accumulation linked to drainage networks, rainfall, solar radiation, and certain specific indices obtained using remote sensing techniques, such as Normalized Difference Vegetation Index (NDVI) or Clay Minerals Index (CMI).

Keywords: soil properties, soil organic matter (SOM), modeling, GIS, environmental variables, remote sensing.

\section{INTRODUCCIÓN}

La materia orgánica del suelo (MOS) configura de manera sustancial las propiedades básicas de los suelos y ejerce una importante función hidrológica y geomorfológica al favorecer la agregación del suelo. Permite la formación de complejos arcillo-húmicos que actúan como núcleo de los agregados del suelo (Lal et al, 1994), aumentando su porosidad y, con ella, la infiltración y percolación de fluidos. En consecuencia, hace incrementar la capacidad de retención de agua en el suelo (Brady, 1984), al tiempo que disminuye la escorrentía y el riesgo de erosión (Van Beers, 1980). A su vez, los procesos erosivos afectan directamente a la movilización de stocks de carbono en el suelo (Martínez-Mena et al., 2012; Nadeu et al., 2012; Boix-Fayos et al., 2015), cuya incidencia en el cambio climático global ha sido ampliamente reseñada (Albaladejo et al., 2013). También resulta especialmente relevante el comportamiento del carbono orgánico (CO), asociado a la MOS, en relación con su manejo y tratamiento, y con su influencia en los servicios ecosistémicos (MEA, 2005). En relación con el estudio del ciclo global de carbono y sus implicaciones en el calentamiento global (Jones et al., 2005), existe una extensa literatura científica al respecto. Numerosos trabajos publicados en este sentido consideran fundamental el conocimiento de la distribución del carbono orgánico y coinciden en afirmar que los stocks de carbono en el suelo son mayores que las de la atmósfera y la biosfera en conjunto (Grace, 2004).

La posibilidad de estimar valores de MOS espacialmente distribuidos puede contribuir a la toma de decisiones en la gestión de estos recursos y minimizar sus efectos perniciosos sobre el medioambiente. Con el fin de avanzar en una cartografía de suelos más detallada a lo largo de los últimos años se han elaborado diferentes técnicas para predecir, sobre grandes extensiones (de aquí en adelante, regionales), el valor de una variable determinada del suelo. En los primeros 1980s se abordó la estimación espacialmente distribuida de variables del suelo mediante la aplicación de técnicas de kriging y cokriging (McBratney et al., 1981), o Regresiones Geográficamente Ponderadas (GWR) (Wang et al., 2013; Wang et al., 2014; 
Zeng et al., 2016), basadas fundamentalmente en la dependencia espacial de las variables de estudio. La utilidad de estas técnicas es mayor para áreas de extensión media y con muestreos bastante intensivos y regulares (Chen et al. 2000; Fox and Sabbagh 2002). En una estimación de carácter regional, con muestras ampliamente dispersas, la información proporcionada por la posible autocorrelación espacial entre muestras puede ser muy reducida (Burrough et al., 1997; Western et al., 2004).

Como la densidad de medidas no es habitualmente muy alta a escala regional, se requiere el uso de otros métodos para predecir los valores de las variables del suelo de manera sencilla y asequible (Ließ et al., 2012). Una alternativa particularmente efectiva es la modelización de la relación entre la variable de interés en el suelo y las variables ambientales de las que se dispone de información espacialmente distribuida (Gessler et al., 1995; Brus y De Gruijter, 1997; McKenzie y Ryan, 1999; Thompson et al., 2001; McBratney et al., 2003; Bou Kheir et al., 2010). La importancia de dicha relación en el proceso de elaboración de la cartografía digital de suelos ha sido ya reseñada por diversos autores mediante el uso de métodos estadísticos aplicados a la predicción espacial de las propiedades del suelo (Minasny et al., 2008; Dobos and Hengl, 2009; Bou Kheir et al., 2010; Greve et al., 2012). El resultado son productos cartográficos con información continua obtenidos mediante modelos estadísticos de las propiedades del suelo en función de las variables ambientales que influyen en su formación y distribución espacial.

Por otra parte, las técnicas de teledetección proporcionan volúmenes masivos de datos cuantitativos georreferenciados, que permiten una visión general de grandes superficies, y valores espectrales que presentan buenas correlaciones con las coberturas de los usos del suelo, además de poseer una capacidad de actualización temporal muy dinámica (BenDor, 2002). La evaluación, caracterización y determinación de las propiedades del suelo, mediante la utilización de datos procedentes de sensores remotos ha sido extensamente aplicada durante los últimos años (Rawls et al., 2004; Vrieling, 2006; Lagacherie et al., 2012; Poggio et al., 2013; Mirzaee et al, 2016). La capacidad de la espectrometría en condiciones de laboratorio está demostrada para la predicción de importantes propiedades del suelo (Schulten y Schnitzer, 1997; ViscarraRossel et al., 2006; Ben-Dor et al., 2009). Sin embargo, su aplicación directa a la superficie terrestre no está exenta de ciertas dificultades y limitaciones, lo que hace aconsejable, en este caso, enfocar su uso a la obtención de índices específicos relacionados con las variables ambientales.

En el presente estudio se explota la capacidad de los SIG para gestionar grandes volúmenes de información ambiental (datos de campo y de teledetección), en la que cada muestra de la base de datos se asocia con los caracteres e índices ambientales correspondientes al lugar de muestreo. Relacionando los valores de la MOS con los de dichas variables ambientales, utilizadas como predictores, se construye un modelo de regresión para predecir el contenido de materia orgánica. El efecto de estas variables sobre el modelo es interesante por las conclusiones que se pueden extraer de las distintas implicaciones que ejercen los patrones geoecológicos sobre la génesis y distribución espacial de la MOS.

\section{II. ÁREA DE ESTUDIO Y CONDICIONES AMBIENTALES}

El área de estudio elegida se centra en la Región de Murcia (11.313 km²) (INE, 2013), encuadrada dentro de la cuenca del Río Segura (18.208 km²), CHS, 2013) en el Sureste de 
España) (Figura 1). En conjunto, se trata de una superficie bastante accidentada por la presencia de numerosas sierras, pertenecientes a las Cordilleras Béticas, que se alinean en dirección ENE-WSW con altitudes a menudo superiores a los $1.000 \mathrm{~m}$. Entre ellas se intercalan una serie de valles, cubetas, llanuras y altiplanos, que en conjunto configuran una topografía contrastada y un territorio singular de gran variedad paisajística. La zona de estudio participa en su mayor parte de las características de un clima mediterráneo semiárido (Capel-Molina, 2000), con una precipitación media de la cuenca en torno a $375 \mathrm{~mm} / \mathrm{año}$, con valores medios anuales de $472 \mathrm{~mm}$ en cabecera y de $317 \mathrm{~mm}$ en zonas próximas a la desembocadura, y una gran irregularidad pluviométrica estacional e interanual, con sequías prolongadas y lluvias a menudo torrenciales.
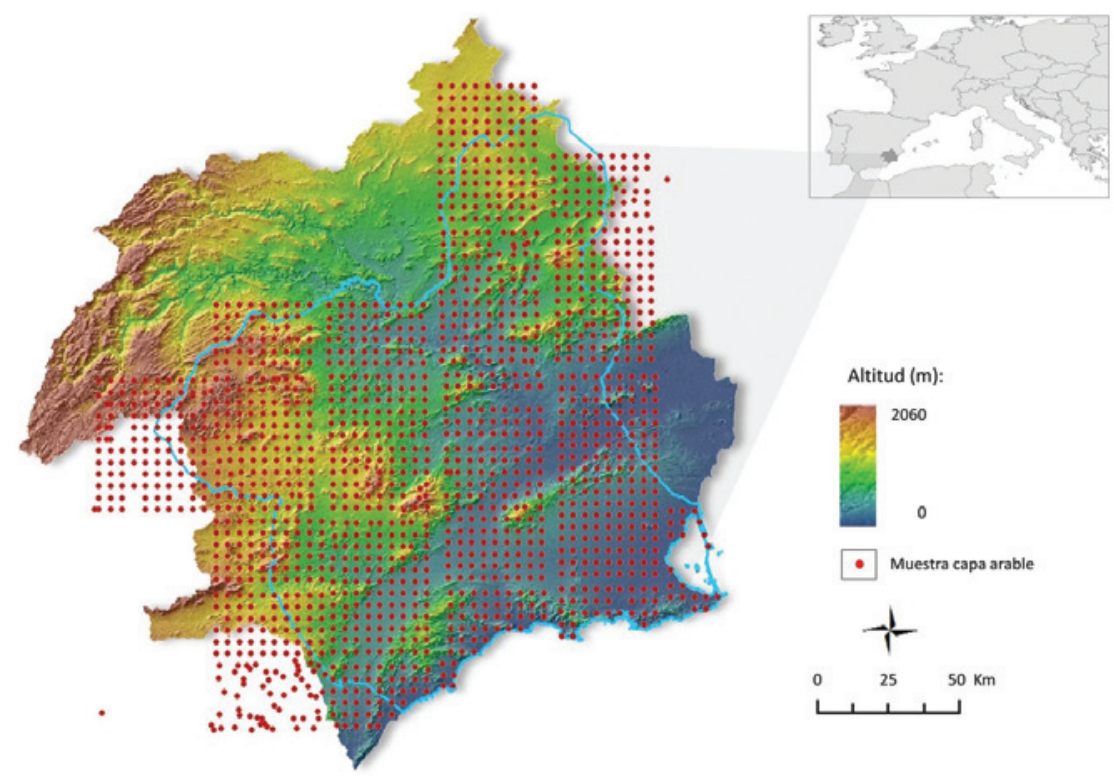

Esta área recibe una fuerte insolación anual, con un promedio de 2.800 horas de sol al año, un máximo en julio (340 h) y un mínimo en diciembre (160 h), según datos del observatorio de Alcantarilla, próximo a la ciudad de Murcia (INM, 2004; Alonso-Sarria, 2007). Por el carácter accidentado del relieve regional, las variaciones locales en la radiación recibida son notables. Sólo en las tierras más llanas (llanura litoral, depresiones pre-litorales y altiplanicies) los contrastes disminuyen y la homogeneidad es mayor. Estimaciones basadas en modelos teóricos de terreno han dado valores medios de $5.023 \mathrm{kWh} / \mathrm{m}^{2} /$ año, valores que superan los 5.500 e incluso los 6.000 en solana y descienden en la umbría, especialmente en las zonas de mayor altitud (Conesa-García y Alonso-Sarría, 2006). 
La desertificación y la pérdida de suelo por erosión se presentan como unas de las causas más importantes de la degradación del suelo en este territorio, ya que implica la pérdida de sus principales componentes físicos, químicos y biológicos (Boix-Fayos et al., 2005). Según datos del Inventario Nacional de Erosión de Suelos, elaborado por el Ministerio de Medio Ambiente (MMA, 2002-2012), la erosión media del suelo en la Cuenca del río Segura se sitúa en torno a la media española, 24,53 ton/ha/año, equivalentes a un rebajamiento de casi $2 \mathrm{~mm}$ anuales. En esta cuenca existe una gran diversidad de suelos originados por la acción de diferentes procesos asociados a la influencia de múltiples factores ambientales. Entre ellos destacan las condiciones climáticas, la litología y el relieve, que condicionan en gran medida la erosión y los procesos edáficos (Álvarez Rogel et al., 2001).

\section{MATERIAL Y MÉTODOS}

\section{III.1. Datos analíticos del Proyecto LUCDEME}

La información empírica sobre el contenido de la MOS ha sido obtenida a partir de datos del Proyecto de Lucha contra la Desertificación en el Mediterráneo (LUCDEME) (ICONA, 1986), elaborado a comienzos de los años ochenta y primeros de los noventa. De este trabajo se obtiene una base de datos de los análisis de los muestreos de campo correspondientes a determinaciones analíticas de la capa arable, relacionada con la capa más superficial del suelo (0-20 cm aproximadamente), tanto en suelos de cultivo como en suelos no cultivados. Los datos forman un total de 1570 muestras distribuidas en una malla regular de $3 \times 3 \mathrm{Km}$, de las cuales, 1126 puntos entran dentro de la zona de estudio (áreas de la Región de Murcia dentro de la Cuenca del Segura) (Figura 1).

\section{III.2. Variables ambientales modelizadas}

Los datos utilizados para los procesos de modelización se agrupan en un una serie de capas de información georreferenciadas, y aunque alguna de ellas no pueden definirse como variables ambientales (índices elaborados a partir de señales espectrales de sensores remotos), se pueden relacionar indirectamente con las características del suelo. De este modo, se mantiene esta definición para homogeneizar toda la información tratada en los análisis de SIG y tratamientos estadísticos.

Estas variables se pueden estructurar en una serie de conjuntos de datos, que son descritos en Variables topográficas e hidrológicas, obtenidas a partir del Modelo Digital de Elevaciones (MDE) (1) (NASA - METI, 2013). A partir de (1) se han calculado: Pendientes (2), (Burrough and McDonnell, 1998). La curvatura que se calcula de manera análoga a (2) (Moore et al., 1991). La curvatura se puede descomponer en Curvatura del perfil (3) y curvatura perpendicular (4) (Zeverbergen and Thorne, 1987), y finalmente la Acumulación de flujo (5) que representa el número de píxeles de la cuenca vertiente a un píxel en particular del MDE (Tarboton et al., 1991). Tipos de suelos y litología con información edafológica y litológica procede de diferentes fuentes. Mapa de suelos del proyecto LUCDEME (E. 1:100.000) (6) la Fase Salina (7), identificada como áreas cuya conductividad eléctrica $>2 \mathrm{mmhos} / \mathrm{cm}$. Mapa de litología (8) realizado a partir de los datos del Mapa Geológico Nacional 1:50.000 (MAGNA). Variables 
climáticas, como precipitación $(\mathrm{mm})(\mathbf{9})$ y la de temperatura $\left({ }^{\circ} \mathrm{C}\right)(\mathbf{1 0})$ para la zona de estudio (LBI, 2013), y diversas variables relacionadas con la radiación solar, Radiación solar directa (11) (Fu and Rich, 2000), (12) la radiación solar difusa (Fu and Rich, 2000), y la Duración de la radiación solar directa (13), indicada en horas de insolación directa para cada uno de los píxeles. Variables complementarias obtenidas mediante Teledetección, se han utilizado imágenes del satélite Landsat para obtener información adicional, mediante el uso de dos fechas (14/02/2009 y 24/07/2009). Estas imágenes corresponden a dos periodos estacionales diferentes del mismo año, uno estival y otro invernal. De esta forma se minimiza el posible sesgo estacional. Para cada periodo se estimó el Índice de Vegetación de Diferencia Normalizada (NDVI) (14 y 15, datos para invierno y verano, respectivamente) (Townshend et al, 1985). También se han aplicado una serie de algoritmos sobre las imágenes seleccionadas para obtener indicadores mineralógicos (Sabins, 1981; Crosta et al., 2003), mediante combinación de las siguientes funciones estandarizadas "Clay Minerals" (minerales de arcilla, CMI) (16 y 19), "Ferrous Minerals" (minerales ferrosos, FMI) (17 y 20) y "Iron Oxide" (óxidos de hierro, IOI) (18 y 21). Para cada una de las funciones se han realizado los cálculos correspondientes a la estación de verano (Julio) e invierno (Febrero).

A continuación (Tabla 1) se exponen los valores resumen de cada una de las variables expuestas:

Tabla 1

DESCRIPCIÓN DE LA REPRESENTATIVIDAD DE LOS VALORES DE LAS VARIABLES AMBIENTALES RASTER Y SU ESTADÍSTICO DESCRIPTIVO CON EL CÁLCULO DEL RANGO, MEDIA Y DESVIACIÓN ESTÁNDAR

\begin{tabular}{|c|c|c|c|c|c|c|}
\hline Id & Variable & Descripción valores mínimos & Descripción valores máximos & Rango & Media & $\begin{array}{c}\text { Desv. } \\
\text { Estándar }\end{array}$ \\
\hline (1) & DEM & Altitud nivel mar & Altitud absoluta & $0-2057(\mathrm{~m})$ & 612 & \pm 407 \\
\hline (2) & Slp & Superficies llanas & Relieve escarpado & $0-79\left(^{\circ}\right)$ & 7,5 & $\pm 7,7$ \\
\hline (3) & $\mathrm{CuP}$ & Curvatura Convexa & Curvatura Cóncava & $-18-18$ & 0,008 & $\pm 0,27$ \\
\hline (4) & CuPP & Curvatura Cóncava & Curvatura Convexa & $-20-22$ & 0,008 & $\pm 0,41$ \\
\hline (5) & FAc & Baja Acumulación de flujo & Alta Acumulación de flujo & $0-17$ (pixel) $*$ & 1,6 & $\pm 1,8$ \\
\hline (9) & Pr & Precipitación mínima & Precipitación máxima & $250-771(\mathrm{~mm})$ & 390 & \pm 84 \\
\hline (10) & Tmp & Bajas temperaturas & Altas temperaturas & $8,2-18,4\left({ }^{\circ} \mathrm{C}\right)$ & 15,1 & $\pm 2,07$ \\
\hline (11) & RaDir & Baja radiación directa & Alta radiación directa & $0,001-2,258 * *$ & $1,36 *$ & $\pm 0,12$ \\
\hline (12) & RaDif & Baja radiación difusa & Alta radiación difusa & $0,083-0,514 * *$ & $0,28 *$ & $\pm 0,01$ \\
\hline (13) & RaDur & Escasa duración de radiación & Elevada duración de radiación & $40-4360(\mathrm{~h})$ & 4,03 & $\pm 0,27$ \\
\hline (14) & NDVIfeb & Suelos artificiales - agua & Abundante vegetación & $-1-1$ & 0,05 & $\pm 0,19$ \\
\hline (15) & NDVIjul & Suelos artificiales - agua & Abundante vegetación & $-1-1$ & 0,03 & $\pm 0,13$ \\
\hline (16) & CMIfeb & Baja alteración minerales CMI & Alta alteración minerales CMI & $0-3,5$ & 1,21 & $\pm 0,97$ \\
\hline (17) & FMIfeb & Baja alteración minerales FMI & Alta alteración minerales FMI & $0-3$ & 0,83 & $\pm 0,75$ \\
\hline (18) & IOIfeb & Baja alteración minerales IOI & Alta alteración minerales IOI & $0-1,5$ & 0,31 & $\pm 0,28$ \\
\hline (19) & CMIjul & Baja alteración minerales CMI & Alta alteración minerales CMI & $0-3,5$ & 1,21 & $\pm 0,92$ \\
\hline (20) & FMIjul & Baja alteración minerales FMI & Alta alteración minerales FMI & $0-3$ & 0,95 & $\pm 0,76$ \\
\hline (21) & IOIjul & Baja alteración minerales IOI & Alta alteración minerales IOI & $0-1,5$ & 0,39 & $\pm 0,34$ \\
\hline
\end{tabular}




\section{III.3. Propuesta de un modelo predictivo para la estimación de la MOS a escala regional}

El objetivo de este trabajo se centra en la variación de la interacción que se producen entre las variables ambientales seleccionadas y los valores obtenidos en los distintos modelos de la MOS. Los procesos de modelización, que se describen más adelante, basados en técnicas estadísticas ampliamente utilizadas y consensuadas se muestran como elementos que nos permiten analizar los efectos descritos por las principales variables ambientales que forman parte de estos modelos predictivos a escala regional.

Estos cálculos se han realizado mediante distintos algoritmos elaborados a través de ArcGIS v.10 (ESRI, Redlands, USA), en las que a partir de las coordenadas de las muestras de la base de datos se obtuvieron los valores de las 21 variables ambientales para píxeles de $400 \mathrm{~m}$ de resolución. Los valores de materia orgánica disponibles para cada punto fueron relacionados en una tabla con los correspondientes a las variables ambientales seleccionadas. Dicha tabla se utilizó como input para el lenguaje de análisis estadístico ' $\mathrm{R}$ ' ( $\mathrm{R}$ Core Team, 2013), con el que se ensayaron modelos estadísticos de regresión, en el que los predictores son capas de SIG, y pueden representarse fácilmente mediante álgebra de mapas (PérezCutillas, 2013).

Los datos analíticos de la MOS corresponden a los 1.126 puntos de muestreo del proyecto LUCDEME en la zona de estudio. Para obtener cada modelo en función de las variables ambientales se aplicó la regresión lineal 'paso a paso hacia adelante' utilizando el Criterio de Información de Akaike (AIC) (1974). Este método ofrece una medida relativa de la pérdida de información cuando un determinado modelo es utilizado para describir la realidad, de modo que finalmente permite seleccionar el modelo que más se ajusta a los datos reales.

Como la respuesta frente a las variables cuantitativas puede ser no lineal, para todas ellas se utilizó en los modelos términos cuadráticos. Por ejemplo, para la precipitación no se incluyó en la construcción de los modelos sólo la variable precipitación sino también su cuadrática precipitación ${ }^{2}$. De esta forma el modelo puede ajustar fácilmente diversas formas curvas.

\section{RESULTADOS}

\section{IV.1. Principales variables ambientales presentes en los modelos de MOS}

Las variables que de manera más continuada se repiten entre los modelos predictivos con los mejores valores de AIC corresponden a la 'Pendiente' [Slp], 'Curvatura del perfil' [CuP], 'Curvatura perpendicular' [CuPP], 'Acumulación de flujo' [FAc], 'Temperatura' [Tmp], 'Radiación Difusa' [RaDif], 'Índice mineralógico de óxidos de hierro' [IOI], 'Índice mineralógico de arcilla' [CMI], 'Índice mineralógico ferroso' [FMI], 'Índice de vegetación' [NDVI] y 'Taxonomía del Suelo LUCDEME' [SLu].

La Figura 2 muestra la representación espacial para la estimación de la MOS para el modelo de mejor índice AIC. 


\section{Figura 2 \\ ESTIMACIÓN DEL CONTENIDO DE MATERIA ORGÁNICA ELABORADA MEDIANTE VARIABLES AMBIENTALES. EXPRESADA EN \% DE CONTENIDO DE MATERIAL, EN TONOS OSCUROS LOS VALORES MÁS ALTOS Y EN CLARO LOS MÁS BAJOS}

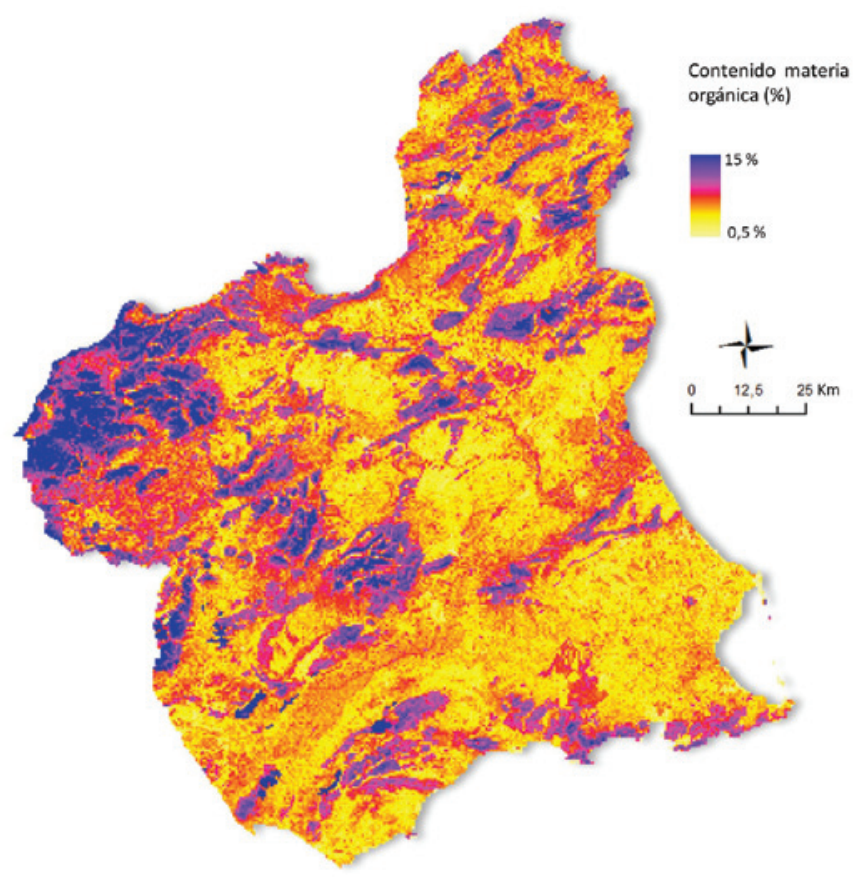

\section{IV.2. Influencia y efecto de las variables ambientales en el contenido de MOS}

Las figuras 3 y 4 muestran una visión de conjunto de cómo influyen las distintas variables ambientales en la predicción de la MOS.

Entre los aspectos topográficos e hidrológicos considerados, intervienen en el modelo de MOS las variables [Slp], [CuP], [CuPP] y [FAc]. El principal efecto que ejerce la variable [Slp] en el modelo es un incremento del porcentaje de materia orgánica asociado al aumento de la pendiente topográfica. En [CuP] las formas convexas tienen un contenido de la MOS más elevado, mientras que en las superficies cóncavas del perfil de máxima pendiente, dicho contenido desciende. Para [CuPP] sucede lo contrario, es decir, se producen mayores concentraciones de MOS en las superficies cóncavas de los perfiles perpendiculares a la máxima pendiente. Por último, la variable [FAc] presenta un efecto cuadrático, con estabilidad del contenido de MOS en las zonas con menor área de drenaje e incremento lineal a partir de un umbral de acumulación de flujo.

La variable [Tmp] describe un incremento en los niveles de MOS conforme aumentan las temperaturas, proceso que tiende a estabilizarse cuando la temperatura media anual se sitúa en torno a los $17-18^{\circ} \mathrm{C}$. Por su parte, el contenido de materia orgánica disminuye con un aumento de la [RaDif] estabilizándose a partir del umbral 0,25 MWh/m² - año. 

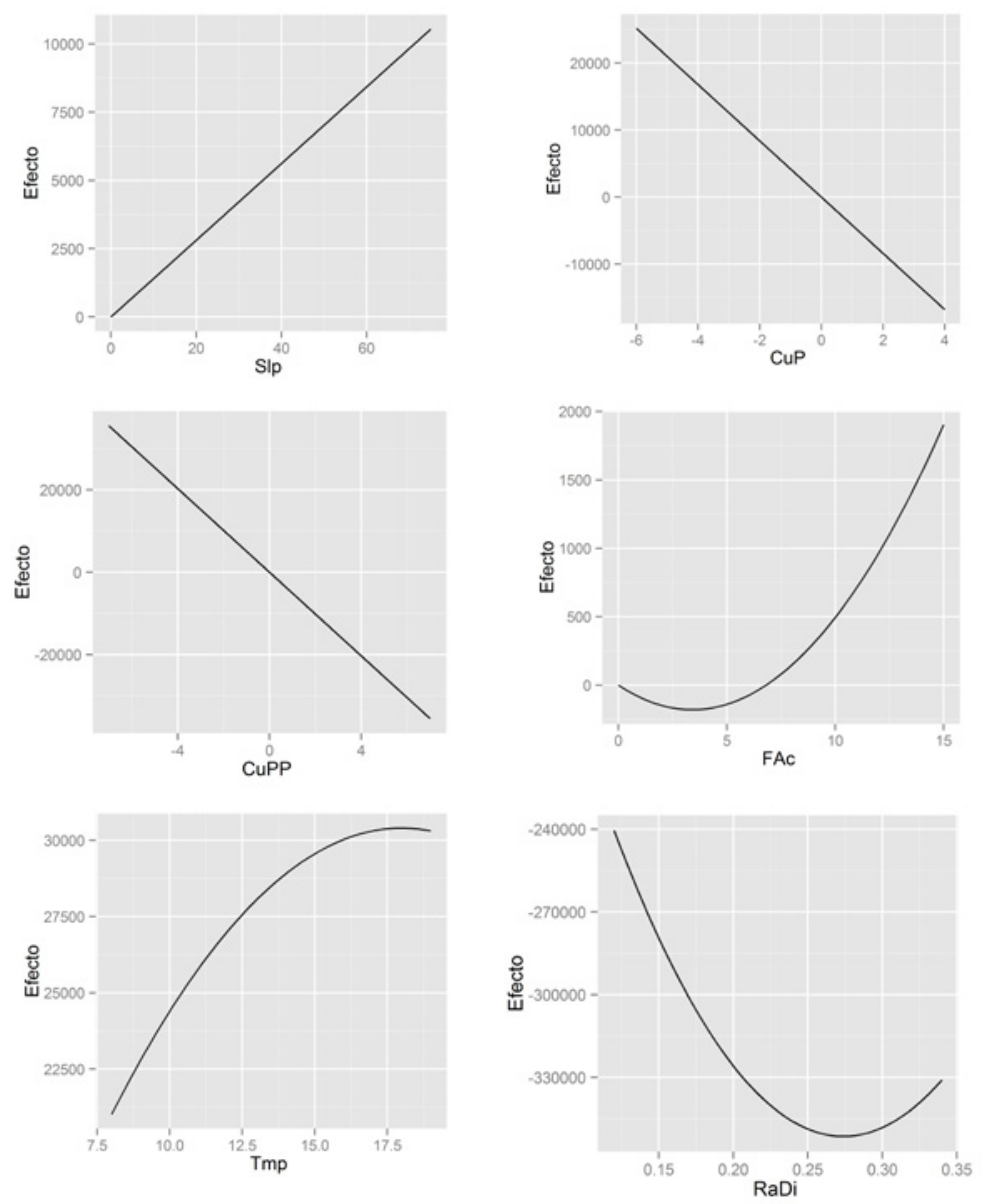

El mayor número de variables que influyen en la estimación de la MOS se obtiene por teledetección. El índice mineralógico [IOI], tanto para febrero y como para julio, se corresponde con una clara reducción del contenido de materia orgánica. Esta disminución se hace patente a medida que los elementos con contenido en hierro detectados por el sensor pasan a valores altos de alteración mineralógica, al igual que sucede con la variable [CMIjul] para las zonas detectadas en niveles de alteración de minerales arcillosos. Por su parte [FMIjul] denota un efecto contrario, con un aumento del contenido en materia orgánica a medida que se hacen más evidentes los signos de alteración de los minerales ferrosos. Finalmente, [NDVIjul] también muestra un aumento del contenido de MOS acorde con el incremento de los valores de respuesta fotosintética, asociada a la presencia de masas de vegetación. 

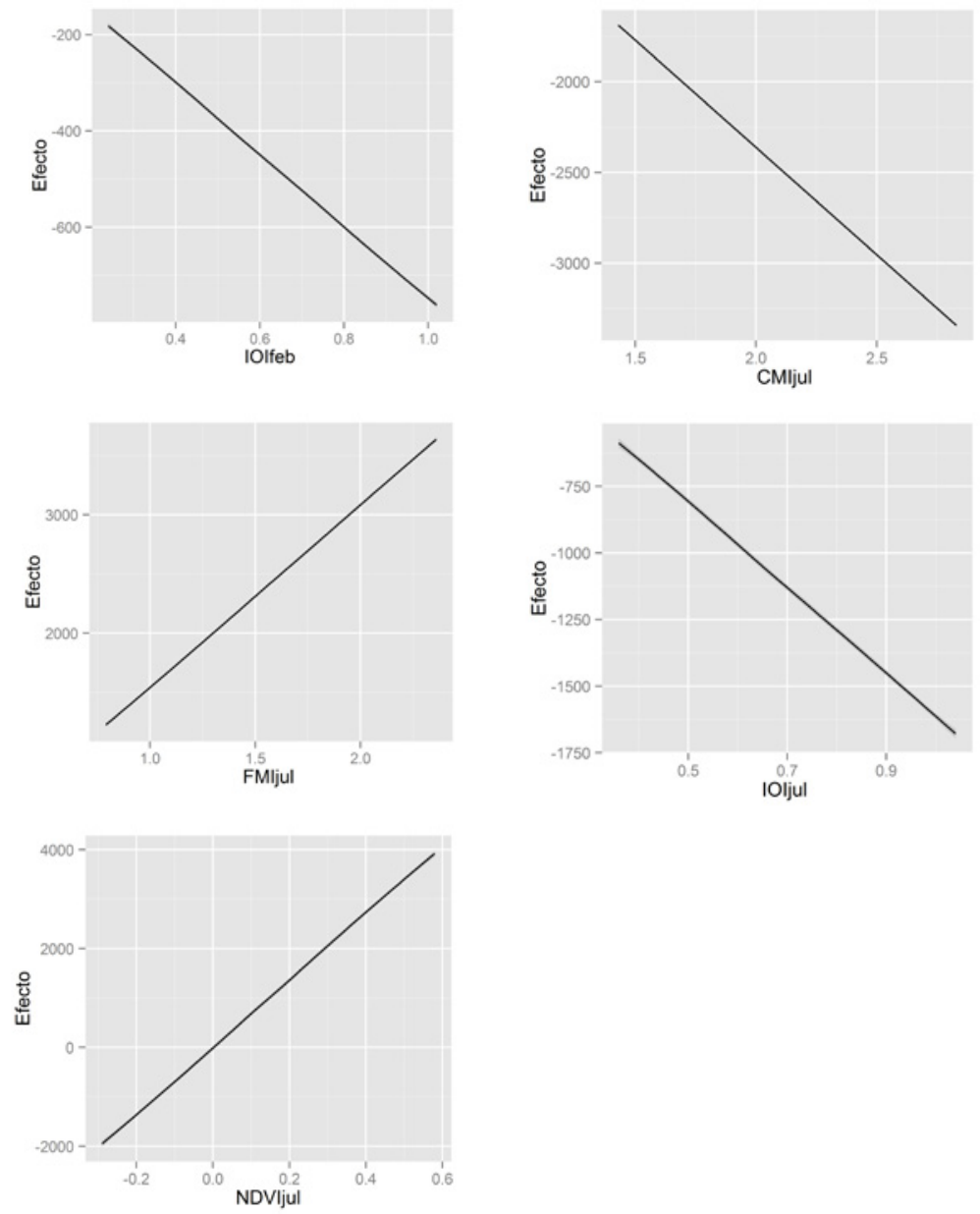

Los efectos observados para los coeficientes de las variables cualitativas presentes en el modelo se muestran a través del efecto de los diferentes niveles de la variable Suelo [SLu], que se dan en relación a la clase 'Arenosoles álbicos', a la que se atribuye arbitrariamente el nivel 0 o de referencia. Las áreas donde el suelo se encuentra en Fase Salina registran un aumento, aunque bastante moderado, del contenido de materia orgánica. En el caso de la variable (SLu), todos los niveles muestran un aumento de los índices de la MOS respecto a la clase de referencia, pero son las superficies de 'Litosoles' y la combinación de estos con la clase 'Xerosoles cálcicos' las que tienen valores más altos. Importantes son también los 'Fluvisoles calcáricos', que alcanzan niveles significativos de relación con la MOS. 


\section{DISCUSIÓN Y CONCLUSIONES}

Se han publicado recientemente diversos trabajos que proporcionan modelos eficientes de predicción del contenido de materia orgánica en las capas superficiales del suelo, relacionando el carbono orgánico con atributos extraídos de un MDE (pendiente, curvaturas,...) y de mapas de suelos (Meersmans et al., 2009; Schwanghart and Jarmer, 2011; Doetterl et al., 2013). Desde un punto de vista conceptual, y en base a estos estudios, se han propuesto los cálculos para la estimación del contenido de la MOS a partir de variables ambientales El principal objetivo de esta información es servir de datos de entrada para modelizar procesos a escala regional y en su estado actual parece adecuado para ello, aunque sea susceptible de posteriores mejoras.

Un modelo correlacional y observacional como el que aquí se ha desarrollado debe interpretarse con cautela. Los modelos de regresión pueden sugerir procesos subyacentes pero si no provienen de una aproximación experimental difícilmente permiten hablar de causalidad. Más aún cuando las variables ambientales están fuertemente correlacionadas entre sí, como es nuestro caso, es normal que dos variables muy correlacionadas que tienen independientemente efectos similares muestren en la regresión coeficientes de signo contrario por efecto 'compensatorio' (una de las dos variables es redundante). AIC tiende a seleccionar a veces modelos excesivamente complejos en esas situaciones.

Respecto al efecto que muestran las variables, se observa en el grupo de las variables topográficas que tienen una notable influencia en la predicción del contenido de la MOS (Berhe, 2012; Doetterl et al., 2012; Wang et al., 2014), y muy especialmente la pendiente (Slp) y las curvaturas (CuP y CuPP) del terreno. En los tres casos existe una relación directa con la disminución de la MOS en las áreas llanas o de escasa pendiente. Es probable que este efecto esté fundamentalmente asociado a la mayor degradación del suelo por la ocupación mayoritaria de los terrenos llanos por la agricultura, mientras que los contenidos altos en MOS suelen registrarse en zonas más escarpadas, al hallarse éstas ocupadas por vegetación natural. Esta relación directa entre vegetación y la predicción de la MOS se observa de igual manera para las variables NDVIjul (Paustian et al, 1997 y Mishra et al., 2010) y FAc. En el primer caso se constata el consabido aumento del contenido de materia orgánica de acuerdo con una mayor ocupación por parte de la vegetación natural. Existen, no obstante, ciertas salvedades, ya que algunos usos agrícolas como los cultivos hortícolas pueden ofrecer un alto valor de NDVI que inducen al modelo a sobrestimar los niveles de MOS para estas zonas. Respecto a la variable FAc, cuyos valores más altos representan las zonas de mayor acumulación de flujo, en estos ambientes semiáridos alcanza su máximo exponente en las áreas con balances hídricos más favorables, donde la vegetación natural tiene un mayor desarrollo.

La relación entre la MOS y temperatura es contra intuitiva, ya que en un medio hídricamente deficitario, como el estudiado, parece contradictorio que a mayor temperatura se incremente la materia orgánica del suelo. Del mapa de la Figura 2 se observa que los mayores contenidos predichos de materia orgánica se dan en las zonas montañosas, si bien la altitud, negativamente relacionada con la temperatura, no entra en la mayoría de los modelos. La pendiente asume ese papel, donde las zonas de mayor pendiente no sólo albergan la vegetación natural sino que además son representativas de las áreas montaño- 
sas con menor temperatura y mayores precipitaciones. Además el NDVI identifica también las zonas con mayor vegetación en las áreas de balance hídrico más favorable y por tanto el efecto neto de la temperatura debe 'descontar' todas esas relaciones.

También se observa que independientemente de otras variables ambientales, disponer de una cartografía de suelos ayuda a mejorar la predicción con resultados similares a los expuestos por Zhang et al. (2012), según los cuales la introducción de las variables categóricas, como los tipos de suelos, mejora la precisión de modelos en la predicción de la MOS.

Finalmente, quedaría por dilucidar por qué determinadas variables de teledetección relacionadas con la composición mineralógica del terreno contribuyen a la predicción de la MOS. Este asunto debe ser estudiado en detalle y contrastar si las ratios espectrales se pueden relacionar con otros atributos ecosistémicos, que a priori tuviesen más relación con la formación y acumulación de materia orgánica. No obstante es interesante explorar si los índices mineralógicos se relacionan con la fertilidad del suelo.

\section{REFERENCIAS BIBLIOGRÁFICAS}

AKAIKE, H. (1974). «A new look at the statistical model identification». IEEE Transactions on Automatic Control, $\mathrm{n}^{\mathrm{o}}$ 19, 716-723.

ALBALADEJO, J., ORTIZ, R., GARCIA-FRANCO, N., NAVARRO, A.R., ALMAGRO, M., PINTADO, J.G. y MARTÍNEZ-MENA, M. (2013). «Land use and climate change impacts on soil organic carbon stocks in semi-arid Spain». Journal of Soils and Sediments, $\mathrm{n}^{\mathrm{o}} 13,265-277$

ALONSO-SARRÍA, F. (2007). Atlas Global de la Región de Murcia. La Verdad - CMM S.A. Murcia. http://www.atlasdemurcia.com/index.php/secciones/1/el-clima/3/

ÁlVAREZ ROGEL, J., ORTIZ SILLA, R. y ALCARAZ ARIZA, F. 2001. «Edaphic characterization and soil ionic composition influencing plant zonation in a semiarid Mediterranean salt marsh». Geoderma, $\mathrm{n}^{\circ}$ 99, 81-98.

BEN-DOR, E. (2002). «Quantitative remote sensing of soil properties». Advances in Agronomy, $\mathrm{n}^{\circ} 75,173-243$.

BEN-DOR, E., CHABRILlAT, S., DEMATTÊ, J.A.M., TAYLOR, G.R., HILL, J., WHITING, M.L. y SOMMER,S. (2009). «Using Imaging Spectroscopy to study soil properties». Remote Sensing of Environment, $\mathrm{n}^{\circ} 113,38-55$.

BERHE, A.A.(2012). «Decomposition of organic substrates at eroding vs depositional landform positions». Plant and Soil, $\mathrm{n}^{\circ}$ 350, 261-280.

BOIX-FAYOS, C., MARTÍNEZ-MENA, M., CALVO-CASES, A., CASTILLO, V. y ALBALADEJO, J. (2005). "Concise review of interrill erosion studies in SE Spain (Alicante and Murcia): Erosion rates and progress of knowledge from the 1980〉s». Land Degradation and Development, $\mathrm{n}^{\circ} 16,517-528$.

BOIX-FAYOS, C. MARTÍNEZ-MENA, M., PÉREZ CUTILLAS, P., DE VENTE, J., BARBERÁ, G.G., MOSCH, W., NAVARRO CANO, J.A., GASPAR, L. y NAVAS, A. (2015). «Carbon redistribution by erosion processes in an intensively disturbed catchment». Catena (en prensa). 
BOU KHEIR, R., GREVE, M.H., BØCHER, P.K., GREVE, M.B., LARSEN, R. y MCCLOY, K.(2010). «Predictive mapping of soil organic carbon in wet cultivated lands using classification-tree based models: The case study of Denmark». Journal of Environmental Management, $\mathrm{n}^{\circ}$ 91, 1150-1160.

BRADY, N.C. (1984). The nature and properties of soils. MacMillan Publishing Company. New York.

BRUS, D.J. y DE GRUIJTER, J.J. (1997). «Random sampling or geostatistical modelling? Choosing between design-based and model-based sampling strategies for soil». Geoderma, $\mathrm{n}^{\circ} 80,1-59$.

BURROUGH, P.A., VAN GAANS, P.F.M. y HOOTSMANS, R. (1997). «Continuous classification in soil survey: Spatial correlation, confusion and boundaries». Geoderma, ${ }^{\circ}$ 77, 115-135.

BURROUGH, P.A. y MCDONNELL, R.A. (1998). Principles of geographical information systems. Oxford University Press, Oxford.

CAPEL-MOLINA, J.J. (2000). El clima de la Península Ibérica. Ariel Geografía, Barcelona.

CHEN, F., KISSEL, D.E., WEST, L.T. and ADKINS, W. (2000). Field-scale mapping of surface soil organic carbon using remotely sensed imagery. Soil Science Society of America Journal, $n^{\circ} 64,746-753$.

CONFEDERACIÓN HIDROGRÁFICA DEL SEGURA (CHS).http://www.chsegura.es/chs/ cuenca/resumendedatosbasicos/marcoadministrativo/. Ministerio de Agricultura, Alimentación y Medio Ambiente. 2013.

CLAUSIUS, R. (1850). On the Motive Power of Heat, and on the Laws which can be deduced from it for the Theory of Heat. Poggendorff's Annalen der Physick, LXXIX.

CONESA GARCÍA, C., y ALONSO SARRIA, F.(2006). El Clima de la Región de Murcia. En Conesa García, C. (Ed.), El Medio Físico de la Región de Murcia. Servicio de Publicaciones, Universidad de Murcia. Murcia, 95-127.

CROSTA, A.P., DE SOUZA FILHO, C.R., AZEVEDO, F. y BRODIE, C. (2003). «Targeting key alteration minerals in epithermal deposits in Patagonia, Argentina, using ASTER imagery and principal component analysis». International Journal of Remote Sensing, $\mathrm{n}^{\circ}$ 24, 4233-4240.

DOBOS, E. y HENGL, T. (2009). Soil mapping applications. In: T. Hengl and H.I. Reuter editors, Geomorphometry, concepts, software and applications. Elsevier, New York, 461479.

DOETTERL, S., SIX, J., VAN WESEMAEL, B. y VAN OOST, K. (2012). «Carbon cycling in eroding landscapes: geomorphic controls on soil organic $\mathrm{C}$ pool composition and $\mathrm{C}$ stabilization». Global Change Biology, nº18, 2218-2232.

DOETTERL, S., STEVENS, A., VAN OOST, K., QUINE, T.A. y VAN WESEMAEL, B. (2013). «Spatially explicit regional-scale prediction of soil organic carbon stocks in cropland using environmental variables and mixed model approaches». Geoderma, n ${ }^{\circ}$ 204205, 31-42.

DURY, S.A. (1987). Image Interpretation in Geology. Chapman \& Hall. London.

FAO. (1974). Soil Map of the World. Vol I. 
FOX, G.A. y SABBAGH, G.J. (2002). Estimation of soil organic matter from red and nearinfrared remotely sensed data using soil line Euclidean techniques. Soil Science Society of America Journal, nº 66, 1922-1929.

FELICÍSIMO, A.M. y FERNÁNDEZ-CEPEDAL, G. (1984). «Estimación de la radiación solar incidente en laderas con pendiente y orientación variables». Studia Oecológica, $\mathrm{n}^{\circ}$ 3, 267-283.

FU, P. y P. M. RICH. (2000). The Solar Analyst 1.0 Manual. Helios Environmental Modeling Institute (HEMI), USA.

GEISSER, S. y JOHNSON, W.M. (2006). Modes of Parametric Statistical Inference (Wiley Series in Probability and Statistics). Wiley-Interscience Ed.

GESSLER, P.E., MOORE, I.D., MCKENZIE, N.J. y RYAN, P.J. (1995). «Soil-landscape modelling and spatial prediction of soil attributes». Int. J. Geographical Information Systems, $\mathrm{n}^{\circ}$ 4, 421-432.

GOBIN, A., CAMPLING, P. y FEYEN, J. (2001). «Soil-Landscape Modelling to Quantify Spatial Variability of Soil Texture». Phys. Chem. Earth (B), $\mathrm{n}^{\circ}$ 26, 41-45.

GRACE, J. (2004). «Understanding and managing the global carbon cycle». Journal of Eco$\log y, \mathrm{n}^{\circ} 92,189-202$.

GREVE, M.H., KHEIR, R.B., GREVE, M.B. y BØCHER, P.K. (2012). «Quantifying the ability of environmental parameters to predict soil texture fractions using regression-tree model with GIS and LIDAR data: The case study of Denmark». Ecol. Indic. n 18, 1-10.

ICONA. Proyecto LUCDEME (Lucha contra la Desertificación del Mediterráneo) (1986). Mapa de suelos, escala 1:100.000. ICONA - Ministerio de Agricultura, Pesca y Alimentación.

INSTITUTO NACIONAL DE ESTADÍSTICA (INE). http://www.ine.es/inebmenu/mnu_ entornofis.htm\#1. Ministerio de Economía y Competitividad. 2013.

JENNY, H. 1941. Factors of soil formation: A system of quantitative pedology. McGrawHill, New York.

JONES, C., MCCONNELL, C., COLEMAN, K., COX, P., FALLOON, P., JENKINSON, D. y POWLSON, D. (2005). «Global climate change and soil carbon stocks, predictions from two contrasting models for the turnover of organic carbon in soil». Global Change Biology, n $11,154-166$.

KIDNER, D., DOREY, M. y SMITH D. (1999). «What s the point? Interpolation and extrapolation with a regular grid DEM». Proceedings of the 4th International Conference on GeoComputation.

LAGACHERIE, P., BAILLY, J.S., MONESTIEZ, P. y GOMEZ, C. (2012). «Using scattered hyperspectral imagery data to map the soil properties of a region». European Journal of Soil Science, $\mathrm{n}^{\circ}$ 63, 110-119.

LAL, R., MAHBOUBI, A.A. y FAUSEY, N.R. (1994). «Long-term tillage and rotation effects onproperties of a central Ohio soil». Soil Science Society of America Journal, ${ }^{\circ}$ $58,517-522$.

LBI, (2013). Laboratorio de Biogeografía Informática.http://www.mncn.csic.es/ Menu/Investigacin/Serviciodeapoyoalainvestigacin/LabBiogeografaInformtica/ seccion=1258\&idioma=es_ES.do. Museo Nacional de Ciencias Naturales. CSIC. 
LIEß, M., GLASER, B. y HUWE, B. (2012). «Uncertainty in the spatial prediction of soil texture Comparison of regression tree and Random Forest models». Geoderma, $\mathrm{n}^{\circ} 170$, 70- 79.

LILLESAND, T.M., KIEFER, R.W. y CHIPMAN, J.W. (2004). Remote Sensing and Image Interpretation. Fifth edition. John Wiley \& Sons, Inc., New York.

MARTÍNEZ-MENA, M., LÓPEZ, J., ALMAGRO, M., ALBALADEJO, J., CASTILlO, V., ORTIZ, R. y BOIX-FAYOS, C. (2012). «Organic carbon enrichment in sediments: Effects of rainfall characteristics under different land uses in a Mediterranean area». Catena, $\mathrm{n}^{\circ}$ 94, 36-42.

MCBRATNEY, A.B., WEBSTER, R. y BURGESS, T.M. (1981). «The design of optimal sampling schemes for local estimation and mapping of regionalized variables-I. Theoryand method». Computers and Geosciences, $\mathrm{n}^{\circ}$ 7, 331-334.

MCBRATNEY, A.B., MENDONÇA SANTOS, M.L. y MINASNY, B. (2003). «On digital soil mapping». Geoderma, no 117, 3-52.

MCKENZIE, N.J. y RYAN, P.J. (1999). «Spatial prediction of soil properties using environmental correlation». Geoderma, $n^{\circ} 89,67-94$.

MEERSMANS, J., VAN WESEMAEL, B., DE RIDDER, F. y VAN MOLLE, M. (2009). «Modelling thethree-dimensional spatial distribution of soil organic carbon (SOC) at the regional scale (Flanders, Belgium)». Geoderma, $\mathrm{n}^{\circ}$ 152, 43-52.

MILLENNIUM ECOSYSTEM ASSESSMENT (MEA). (2005). Ecosystems and Human Well-Being: Synthesis. Island Press, Washington.

MINASNY, B., y MCBRATNEY, A.B. (2008). «Regression rules as a tool for predicting soil properties from infrared reflectance spectroscopy». Chemom. Intell. Lab. Syst., $\mathrm{n}^{\circ}$ 94, 72-74.

MINISTERIO DE MEDIO AMBIENTE (MMA) (2002-2012). Inventario Nacional de Erosión de Suelos. http://www.magrama.gob.es/es/biodiversidad/temas/inventariosnacionales/inventarionacional-de-erosion-de-suelos/.

MIRZAEE, S., GHORBANI-DASHTAKI, S., MOHAMMADI, J., ASADI, H. y ASADZADEH, F. (2016). «Spatial variability of soil organic matter using remote sensing data». Catena $\mathrm{n}^{\mathrm{o}} 145,118-127$.

MISHRA, U., LAI, R., LIU, D. y VAN MEIRVENNE, M. (2010). «Predicting the spatial variation of the soil organic carbon pool at a regional scale». Soil Science Society of America Journal, $\mathrm{n}^{\circ} 74$, 906-914.

MOORE, I.D., GRAYSON, R.B. y LANDSON, A.R.(1991). «Digital Terrain Modelling: A Review of Hydrological, Geomorphological, and Biological Applications». Hydrological Processes, $\mathrm{n}^{\circ}$ 5, 3-30.

NADEU, E., BERHE, A.A., DE VENTE, J. y BOIX-FAYOS, C. (2012). «Erosion, deposition and replacement of soil organic carbon in Mediterranean catchments: A geomorphological, isotopic and land use change approach». Biogeosciences, ${ }^{\circ}$ 9, 1099-1111.

NASA - METI. (2013). ASTER GDEM. http://gdem.ersdac.jspacesystems.or.jp/. The Ministry of Economy, Trade and Industry of Japan (METI) and the National Aeronautics and Space Administration (NASA) 
PAUSTIAN, K., LEVINE, E., POST, W.M. y RYZHOVA, I.M. (1997). «The use of models to integrate information and understanding of soil $\mathrm{C}$ at the regional scale». Geoderma, $\mathrm{n}^{\circ}$ 79, 227-260.

POGGIO, L., GIMONA, A. y BREWER, M.J. (2013). «Regional scale mapping of soil properties and their uncertainty with a large number of satellite-derived covariates». Geoderma, no 209-210, 1-14.

R CORE TEAM (2013). R: A language and environment for statistical computing. R Foundation for Statistical Computing, Vienna, Austria. URL http://www.R-project.org/.

RAWLS, W., COSH, M., JACKSON, T. y NEMES, A. (2004). «Use of remotely sensed soil moisture to determine soil hydraulic properties». International Geoscience and Remote Sensing Symposium (IGARSS), $\mathrm{n}^{\circ}$ 4, 2791-2794.

RICH, P. M., DUBAYAH,R., HETRICK, W.A. y SAVING, S.C. (1994). «Using Viewshed Models to Calculate Intercepted Solar Radiation: Applications in Ecology». American Society for Photogrammetry and Remote Sensing Technical Papers, 524-529.

SABINS JR, F.F. (1981). Geological applications and training in remote sensing. CORSE 81: the 1981 conference on remote sensing education, Lafayette, IN : 77-82.

SABINS JR, F.F. (1999). «Remote sensing for mineral exploration». Ore Geology Reviews, $157-183$

SCHULTEN, H.-R. y SCHNITZER, M. (1997). «Chemical model structures for soil organic matter and soils». Soil Science, $\mathrm{n}^{\circ}$ 162, 115-130.

SCHWANGHART, W. y JARMER, T.(2011). «Linking spatial patterns of soil organic carbon to topography - a case study from south-eastern Spain». Geomorphology, ${ }^{\circ} 126$, 252-263.

SHARMA, S.K., MOHANTY, B.P. y ZHU, J. (2006). «Including topography and vegetation attributes for developing pedotransfer functions». Soil Science Society of America Journal, $\mathrm{n}^{\circ} 70,1430-1440$.

TARBOTON, D. G., BRAS, R. L. y RODRIGUEZ-ITURBE, I. (1991). «On the Extraction of Channel Networks from Digital Elevation Data». Hydrological Processes, $\mathrm{n}^{\circ} 5$, 81-100.

THOMPSON, J.A., BELL, J.C. y BUTLER, C.A. (2001). «Digital elevation model resolution: Effects on terrain attribute calculation and quantitative soil-landscape modeling». Geoderma, $\mathrm{n}^{\circ}$ 100, 67-89.

TOWNSHEND, J.R.G., GOFF, T.E. y TUCKER, C.J. (1985). «Multitemporal dimensionality of images of Normalized Difference Vegetation Index at continental scales». IEEE Transactions on Geoscience and Remote sensing, $\mathrm{n}^{\circ} 23,888-895$.

VAN BEERS, W.F.J. (1980). Soils and soil properties. In Drainage Principles and Applications. Vol. I. ILRI. Wageningen.

VRIELING, A. (2006). «Satellite remote sensing for water erosion assessment: A review». Catena, $\mathrm{n}^{\circ} 65,2-18$.

VISCARRA-ROSSEL, R.A., WALVOORT, D.J.J., MCBRATNEY, A.B., JANIK, L.J. y SKJEMSTAD, J.O. (2006). «Visible, near infrared, mid infrared or combined diffuse reflectance spectroscopy for simultaneous assessment of various soil properties». Geoderma, $\mathrm{n}^{\mathrm{o}} 131,59-75$. 
WANG, K., ZHANG, C. y LI, W. (2013). «Predictive mapping of soil total nitrogen at a regional scale: A comparison between geographically weighted regression and cokriging». Applied Geography, n 42, 73-85

WANG, K., ZHANG, C., LI, W., LIN, J. y ZHANG, D.X. (2014). «Mapping soil organic matter with limited sample data using geographically weighted regression». Journal of Spatial Science, ${ }^{\circ}$ 59, 91-106

WESTERN, A.W., ZHOU, S.-L., GRAYSON, R.B., MCMAHON, T.A., BLÖSCHL, G. y WILSON, D.J. (2004). «Spatial correlation of soil moisture in small catchments and its relationship todominant spatial hydrological processes». Journal of Hydrology, $\mathrm{n}^{\circ} 286$, 113-134.

ZHANG, S., HUANG, Y., SHEN, C., YE, H. y DU, Y. (2012). «Spatial prediction of soil organic matter using terrain indices and categorical variables as auxiliary information». Geoderma, $\mathrm{n}^{\circ} 171-172,35-43$.

ZENG, C., YANG, L., ZHU, A.-X., ROSSITER, D.G., LIU, J., LIU, J., QIN, C. y WANG, D. (2016). «Mapping soil organic matter concentration at different scales using a mixed geographically weighted regression method». Geoderma, n 281, 69-82.

ZEVERBERGEN, L.W. y THORNE, C.R. (1987). «Quantitative Analysis of Land Surface Topography». Earth Surface Processes and Landforms, $\mathrm{n}^{\circ}$ 12, 47-56. 
